Proceedings of the Edinburgh Mathematical Society (2007) 50, 173-183 (C)

DOI:10.1017/S0013091505000490 Printed in the United Kingdom

\title{
INCOMPLETE BESSEL FUNCTIONS. I
}

\author{
D. S. JONES
}

Division of Mathematics, University of Dundee, Dundee DD1 4HN, UK

(Received 15 September 2005)

\begin{abstract}
Incomplete Bessel functions of general order are defined. Their recurrence relations, integral representations and asymptotics are derived.
\end{abstract}

Keywords: Bessel functions; incomplete Bessel functions; asymptotics

2000 Mathematics subject classification: Primary 33A40

\section{Introduction}

Incomplete Bessel functions of zero order have been found useful in a number of applications to electromagnetic waves (see, for example, $[\mathbf{1}, \mathbf{3}, \mathbf{5}, \mathbf{8}, \mathbf{9}]$ ).

Definitions of incomplete Bessel functions of general order are introduced below, and some of their properties are obtained. The definitions, which hold for all values of the parameters, are such that the interrelationships between incomplete functions parallel, as far as is feasible, those for standard Bessel functions. They differ therefore from the definitions in the text of [1] ; more information about the differences is at the end of the next section.

\section{Definitions}

The starting point is the definition for the incomplete modified Bessel function, which is based on the modified Bessel function $K_{\nu}(z)$. A suitable definition is

$$
K_{\nu}(z, w)=K_{\nu}(z)-J(z, \nu, w)
$$

where

$$
J(z, \nu, w)=\int_{0}^{w} \mathrm{e}^{-z \cosh t} \cosh \nu t \mathrm{~d} t
$$

When $|\operatorname{ph} z|<\pi / 2$,

$$
K_{\nu}(z)=\int_{0}^{\infty} \mathrm{e}^{-z \cosh t} \cosh \nu t \mathrm{~d} t
$$


and so

$$
K_{\nu}(z, w)=\int_{w}^{\infty} \mathrm{e}^{-z \cosh t} \cosh \nu t \mathrm{~d} t
$$

when $|\operatorname{ph} z|<\pi / 2$. The representation (2.4) explains why the terminology of calling $K_{\nu}(z, w)$ an incomplete modified Bessel function is pertinent.

Observe that $K_{\nu}(z, 0)=K_{\nu}(z)$ and that, as $w \rightarrow-\infty$ with $z$ fixed and $|\operatorname{ph} z|<\pi / 2$, $K_{\nu}(z, w) \rightarrow 2 K_{\nu}(z)$. Also,

$$
K_{-\nu}(z, w)=K_{\nu}(z, w) .
$$

Other incomplete Bessel functions are defined in terms of $K_{\nu}(z, w)$ by the same rules that relate Bessel functions and $K_{\nu}(z)$. Thus,

$$
\begin{aligned}
H_{\nu}^{(1)}(z, w) & =\frac{2}{\pi \mathrm{i}} \mathrm{e}^{-\nu \pi \mathrm{i} / 2} K_{\nu}\left(z \mathrm{e}^{-\pi \mathrm{i} / 2}, w\right), \\
& =H_{\nu}^{(1)}(z)-\frac{2}{\pi \mathrm{i}} \mathrm{e}^{-\nu \pi \mathrm{i} / 2} J(-\mathrm{i} z, \nu, w)
\end{aligned}
$$

and

$$
\begin{aligned}
H_{\nu}^{(2)}(z, w) & =-\frac{2}{\pi \mathrm{i}} \mathrm{e}^{\nu \pi \mathrm{i} / 2} K_{\nu}\left(z \mathrm{e}^{\pi \mathrm{i} / 2}, w\right), \\
& =H_{\nu}^{(2)}(z)+\frac{2}{\pi \mathrm{i}} \mathrm{e}^{\nu \pi \mathrm{i} / 2} J(\mathrm{i} z, \nu, w) .
\end{aligned}
$$

It follows from (2.6), (2.5) and (2.7) that

$$
\begin{aligned}
& H_{-\nu}^{(1)}(z, w)=\mathrm{e}^{\nu \pi \mathrm{i}} H_{\nu}^{(1)}(z, w), \\
& H_{-\nu}^{(2)}(z, w)=\mathrm{e}^{-\nu \pi \mathrm{i}} H_{\nu}^{(2)}(z, w) .
\end{aligned}
$$

In a similar manner,

$$
\begin{aligned}
J_{\nu}(z, w) & =\frac{1}{2}\left\{H_{\nu}^{(1)}(z, w)+H_{\nu}^{(2)}(z, w)\right\} \\
& =J_{\nu}(z)+\frac{1}{\pi \mathrm{i}}\left\{\mathrm{e}^{\nu \pi \mathrm{i} / 2} J(\mathrm{i} z, \nu, w)-\mathrm{e}^{-\nu \pi \mathrm{i} / 2} J(-\mathrm{i} z, \nu, w)\right\}, \\
Y_{\nu}(z, w) & =\frac{1}{2 \mathrm{i}}\left\{H_{\nu}^{(1)}(z, w)-H_{\nu}^{(2)}(z, w)\right\} \\
& =Y_{\nu}(z)+\frac{1}{\pi}\left\{\mathrm{e}^{\nu \pi \mathrm{i} / 2} J(\mathrm{i} z, \nu, w)+\mathrm{e}^{-\nu \pi \mathrm{i} / 2} J(-\mathrm{i} z, \nu, w)\right\}, \\
I_{\nu}(z, w) & =\frac{1}{\pi \mathrm{i}}\left\{\mathrm{e}^{-\nu \pi \mathrm{i}} K_{\nu}(z, w)-K_{\nu}\left(z \mathrm{e}^{\pi \mathrm{i}}, w\right)\right\} \\
& =\mathrm{e}^{-\nu \pi \mathrm{i} / 2} J_{\nu}\left(z \mathrm{e}^{\pi \mathrm{i} / 2}, w\right) \\
& =I_{\nu}(z)+\frac{1}{\pi \mathrm{i}}\left\{J(-z, \nu, w)-\mathrm{e}^{-\nu \pi \mathrm{i}} J(z, \nu, w)\right\} .
\end{aligned}
$$

From (2.12),

$$
I_{-\nu}(z, w)-I_{\nu}(z, w)=I_{-\nu}(z)-I_{\nu}(z)-\frac{2}{\pi} \sin \nu \pi J(z, \nu, w),
$$


whence, by virtue of

$$
I_{\nu}(z)=I_{-\nu}(z)-\left(\frac{2}{\pi}\right) \sin \nu \pi K_{\nu}(z)
$$

and (2.1),

In particular,

$$
K_{\nu}(z, w)=\frac{1}{2} \pi \frac{I_{-\nu}(z, w)-I_{\nu}(z, w)}{\sin \nu \pi}
$$

$$
I_{-n}(z, w)=I_{n}(z, w)
$$

for integer $n$.

Similarly,

$$
H_{\nu}^{(1)}(z, w)=\frac{J_{-\nu}(z, w)-\mathrm{e}^{-\nu \pi \mathrm{i}} J_{\nu}(z, w)}{\mathrm{i} \sin \nu \pi}=\frac{Y_{-\nu}(z, w)-\mathrm{e}^{-\nu \pi \mathrm{i}} Y_{\nu}(z, w)}{\sin \nu \pi}
$$

and

$$
H_{\nu}^{(2)}(z, w)=\frac{\mathrm{e}^{\nu \pi \mathrm{i}} J_{\nu}(z, w)-J_{-\nu}(z, w)}{\mathrm{i} \sin \nu \pi}=\frac{Y_{-\nu}(z, w)-\mathrm{e}^{\nu \pi \mathrm{i}} Y_{\nu}(z, w)}{\sin \nu \pi} .
$$

The book by Agrest and Maksimov [1] starts with several definitions of incomplete Bessel functions and then develops their properties. The definitions hold under the restriction $\mathcal{R}\left(\nu+\frac{1}{2}\right)>0$ and a typical example is

$$
J_{\nu}(w, z)=\frac{2}{\left(\nu-\frac{1}{2}\right) ! \pi^{1 / 2}}\left(\frac{z}{2}\right)^{\nu} \int_{0}^{w} \cos (z \cos \theta) \sin ^{2 \nu} \theta \mathrm{d} \theta
$$

the analogue of the Bessel function $J_{\nu}(z)$. Note that $J_{\nu}(0, z)=0$ and that, depending on the value of $\nu,\left[\partial J_{\nu}(w, z) / \partial w\right]_{w=0}$ is zero or infinite. Later in the book the authors drop the restriction on $\nu$ and take

$$
j_{\nu}(w, z)=\frac{1}{2 \pi \mathrm{i}} \int_{w^{*}}^{w} \mathrm{e}^{z \sinh t-\nu t} \mathrm{~d} t
$$

as the analogue of $J_{\nu}$, where $w^{*}$ is the complex conjugate of $w$. Although $j_{\nu}(0, z)=0$, its derivative with respect to $w$ is totally different from that of $J_{\nu}(w, z)$. So the definitions of (2.17) and (2.18) do not represent the same incomplete Bessel function.

It is characteristic of the other incomplete functions in the came class as $J_{\nu}(w, z)$ in (2.17) to vanish at $w=0$ and to be represented by integrals of similar type. That statement is also true for the incomplete functions related to (2.18). In contrast, the definitions commencing with (2.1) reduce to standard Bessel functions when $w=0$ and are related by rules similar to those satisfied by standard Bessel functions.

\section{Differential equations}

It may be verified by integration by parts that $K_{\nu}(z, w)$ is a solution of the inhomogeneous differential equation

$$
\left\{z^{2} \frac{\partial^{2}}{\partial z^{2}}+z \frac{\partial}{\partial z}-\nu^{2}-z^{2}\right\} K_{\nu}(z, w)=(\nu \sinh \nu w+z \cosh \nu w \sinh w) \mathrm{e}^{-z \cosh w} .
$$


Consequently, $K_{\nu}(z, w)$ is a solution of the homogeneous partial differential equation

$$
\left\{z^{2} \frac{\partial^{2}}{\partial z^{2}}+z \frac{\partial}{\partial z}-\nu^{2}-z^{2}-\frac{\partial^{2}}{\partial w^{2}}+2 \nu \tanh \nu w \frac{\partial}{\partial w}\right\} K_{\nu}(z, w)=0 .
$$

It is evident from (2.12) that $I_{\nu}(z, w)$ also satisfies (3.2). On the other hand, $J_{\nu}(z, w)$, $Y_{\nu}(z, w), H_{\nu}^{(1)}(z, w)$ and $H_{\nu}^{(2)}(z, w)$ are solutions of

$$
\left\{z^{2} \frac{\partial^{2}}{\partial z^{2}}+z \frac{\partial}{\partial z}-\nu^{2}+z^{2}-\frac{\partial^{2}}{\partial w^{2}}+2 \nu \tanh \nu w \frac{\partial}{\partial w}\right\} J_{\nu}(z, w)=0 .
$$

Analogous versions of (3.1) can be derived for the other functions without difficulty but details are omitted.

\section{Recurrence formulae}

Relations connecting different values of $\nu$ can be deduced from the previous sections and the corresponding recurrence formulae for Bessel functions in a straightforward way. For example,

$$
K_{\nu-1}(z, w)+K_{\nu+1}(z, w)=-2 \frac{\partial}{\partial z} K_{\nu}(z, w)
$$

In contrast

$$
K_{\nu-1}(z, w)-K_{\nu+1}(z, w)=-2 \frac{\nu}{z} K_{\nu}(z, w)+\frac{2}{z} \tanh \nu w \frac{\partial}{\partial w} K_{\nu}(z, w) .
$$

The formulae for the other functions are

$$
\begin{aligned}
I_{\nu-1}(z, w)+I_{\nu+1}(z, w) & =2 \frac{\partial}{\partial z} I_{\nu}(z, w) \\
I_{\nu-1}(z, w)-I_{\nu+1}(z, w) & =2 \frac{\nu}{z} I_{\nu}(z, w)-\frac{2}{z} \tanh \nu w \frac{\partial}{\partial w} I_{\nu}(z, w), \\
H_{\nu-1}^{(1)}(z, w)-H_{\nu+1}^{(1)}(z, w) & =2 \frac{\partial}{\partial z} H_{\nu}^{(1)}(z, w), \\
H_{\nu-1}^{(1)}(z, w)+H_{\nu+1}^{(1)}(z, w) & =2 \frac{\nu}{z} H_{\nu}^{(1)}(z, w)-\frac{2}{z} \tanh \nu w \frac{\partial}{\partial w} H_{\nu}^{(1)}(z, w), \\
H_{\nu-1}^{(2)}(z, w)-H_{\nu+1}^{(2)}(z, w) & =2 \frac{\partial}{\partial z} H_{\nu}^{(2)}(z, w), \\
H_{\nu-1}^{(2)}(z, w)+H_{\nu+1}^{(2)}(z, w) & =2 \frac{\nu}{z} H_{\nu}^{(2)}(z, w)-\frac{2}{z} \tanh \nu w \frac{\partial}{\partial w} H_{\nu}^{(2)}(z, w), \\
J_{\nu-1}(z, w)-J_{\nu+1}(z, w) & =2 \frac{\partial}{\partial z} J_{\nu}(z, w), \\
J_{\nu-1}(z, w)+J_{\nu+1}(z, w) & =2 \frac{\nu}{z} J_{\nu}(z, w)-\frac{2}{z} \tanh \nu w \frac{\partial}{\partial w} J_{\nu}(z, w), \\
Y_{\nu-1}(z, w)-Y_{\nu+1}(z, w) & =2 \frac{\partial}{\partial z} Y_{\nu}(z, w), \\
Y_{\nu-1}(z, w)+Y_{\nu+1}(z, w) & =2 \frac{\nu}{z} Y_{\nu}(z, w)-\frac{2}{z} \tanh \nu w \frac{\partial}{\partial w} Y_{\nu}(z, w) .
\end{aligned}
$$


A consequence of (4.1) and (2.5) is

$$
\frac{\partial}{\partial z} K_{0}(z, w)=-K_{1}(z, w)
$$

Likewise, from (4.3) and (2.14),

$$
\frac{\partial}{\partial z} I_{0}(z, w)=I_{1}(z, w)
$$

while, from (4.5) and (2.8),

$$
\frac{\partial}{\partial z} H_{0}^{(1)}(z, w)=-H_{1}^{(1)}(z, w)
$$

The functions $H_{\nu}^{(2)}(z, w), J_{\nu}(z, w)$ and $Y_{\nu}(z, w)$ also satisfy (4.15). The relations (4.13)(4.15) may also be obtained directly from the original definitions.

\section{Integral representations}

One representation of $K_{\nu}(z, w)$ has already been given in (2.4). It holds for $|\mathrm{ph} z|<\pi / 2$ and is based on (2.3). A wider range of $\mathrm{ph} z$ can be obtained by extending (2.3) by analytic continuation so that

$$
K_{\nu}(z)=\int_{0}^{\infty+\mathrm{i} \sigma} \mathrm{e}^{-z \cosh t} \cosh \nu t \mathrm{~d} t
$$

for $|\operatorname{ph} z+\sigma|<\pi / 2$. A simple cross-check of (5.1) is provided by taking $\sigma=-\pi$ and replacing $z$ by $z \mathrm{e}^{\pi \mathrm{i}}$. Rewrite the integral as

$$
\frac{1}{2} \int_{\infty+\pi \mathrm{i}}^{\infty-\pi \mathrm{i}} \mathrm{e}^{z \cosh t-\nu t} \mathrm{~d} t+\frac{1}{2} \int_{-\infty-\pi \mathrm{i}}^{\infty-\pi \mathrm{i}} \mathrm{e}^{z \cosh t+\nu t} \mathrm{~d} t
$$

or

$$
-\pi \mathrm{i} I_{\nu}(z)+\mathrm{e}^{-\nu \pi \mathrm{i}} K_{\nu}(z)
$$

and then the usual formula for $K_{\nu}\left(z \mathrm{e}^{\pi \mathrm{i}}\right)$ is recovered. More generally, agreement for $K_{\nu}\left(z \mathrm{e}^{m \pi \mathrm{i}}\right)$ can be secured by a parallel dissection.

One consequence of (5.1) is that

$$
K_{\nu}(z, w)=\int_{w}^{\infty+\mathrm{i} \sigma} \mathrm{e}^{-z \cosh t} \cosh \nu t \mathrm{~d} t
$$

for $|\mathrm{ph} z+\sigma|<\pi / 2$.

Representations for the other incomplete Bessel functions may now be inferred from the definitions in $\S 2$. Thus,

$$
H_{\nu}^{(1)}(z, w)=\frac{2}{\pi \mathrm{i}} \mathrm{e}^{-\nu \pi \mathrm{i} / 2} \int_{w}^{\infty+\mathrm{i} \sigma} \mathrm{e}^{\mathrm{i} z \cosh t} \cosh \nu t \mathrm{~d} t
$$


for $0<\operatorname{ph} z+\sigma<\pi$ and

$$
H_{\nu}^{(2)}(z, w)=-\frac{2}{\pi \mathrm{i}} \mathrm{e}^{\nu \pi \mathrm{i} / 2} \int_{w}^{\infty+\mathrm{i} \sigma} \mathrm{e}^{-\mathrm{i} z \cosh t} \cosh \nu t \mathrm{~d} t
$$

for $-\pi<\operatorname{ph} z+\sigma<0$. Consequently, for $0<\operatorname{ph} z+\sigma<\pi$,

$$
\begin{aligned}
J_{\nu}(z, w)=\frac{1}{\pi \mathrm{i}} \mathrm{e}^{-\nu \pi \mathrm{i} / 2} & \int_{w}^{\infty+\mathrm{i} \sigma} \mathrm{e}^{\mathrm{i} z \cosh t} \cosh \nu t \mathrm{~d} t \\
& -\frac{1}{\pi \mathrm{i}} \mathrm{e}^{\nu \pi \mathrm{i} / 2} \int_{w}^{\infty+\mathrm{i} \sigma-\mathrm{i} \pi} \mathrm{e}^{-\mathrm{i} z \cosh t} \cosh \nu t \mathrm{~d} t \\
Y_{\nu}(z, w)=-\frac{1}{\pi} \mathrm{e}^{-\nu \pi \mathrm{i} / 2} & \int_{w}^{\infty+\mathrm{i} \sigma} \mathrm{e}^{\mathrm{i} z \cosh t} \cosh \nu t \mathrm{~d} t \\
& -\frac{1}{\pi} \mathrm{e}^{\nu \pi \mathrm{i} / 2} \int_{w}^{\infty+\mathrm{i} \sigma-\mathrm{i} \pi} \mathrm{e}^{-\mathrm{i} z \cosh t} \cosh \nu t \mathrm{~d} t .
\end{aligned}
$$

Furthermore,

$$
I_{\nu}(z, w)=\frac{1}{\pi \mathrm{i}} \mathrm{e}^{-\nu \pi \mathrm{i}} \int_{w}^{\infty+\mathrm{i} \sigma} \mathrm{e}^{-z \cosh t} \cosh \nu t \mathrm{~d} t-\frac{1}{\pi \mathrm{i}} \int_{w}^{\infty+\mathrm{i} \sigma-\mathrm{i} \pi} \mathrm{e}^{z \cosh t} \cosh \nu t \mathrm{~d} t
$$

for $|\operatorname{ph} z+\sigma|<\pi / 2$.

\section{Small argument}

Since the behaviour of Bessel functions for small arguments is well known, it is only necessary to examine the behaviour of $J(z, \nu, w)$ as $z \rightarrow 0$. Expansion of the exponential gives

$$
J(z, \nu, w)=\sum_{n=0}^{\infty} \frac{(-z)^{n}}{n !} \int_{0}^{w} \cosh \nu t \cosh ^{n} t \mathrm{~d} t
$$

Since

$$
\cosh \nu t \cosh ^{n} t=\frac{1}{2^{n}} \sum_{m=0}^{n} \frac{n !}{m !(n-m !) !} \cosh (\nu+n-2 m) t,
$$

the expansion becomes

$$
J(z, \nu, w)=\sum_{n=0}^{\infty}\left(-\frac{1}{2} z\right)^{n} \sum_{m=0}^{n} \frac{\sinh (\nu+n-2 m) w}{m !(n-m !) !(\nu+n-2 m)} .
$$

\section{Large argument}

Rather obviously, the presence of so many parameters in (5.2) makes a determination of its asymptotics non-trivial. As a result, a full discussion will not be attempted here, but will be dealt with in $[\mathbf{7}]$. Instead, the treatment will be limited to what happens when 
$w$ is real and $\sigma=0$. Even with this simplification, the integral exhibits some interesting properties.

The purpose of this section is to consider

$$
K_{\nu}(z, w)=\int_{w}^{\infty} \mathrm{e}^{-z \cosh t} \cosh \nu t \mathrm{~d} t
$$

as $|z| \rightarrow \infty$ with $|\operatorname{ph} z|<\pi / 2$ and $w$ real. It is assumed that $w$ and $\nu$ are kept fixed as $|z| \rightarrow \infty$. Relevant theorems are available in $[\mathbf{2}, \mathbf{6}, \mathbf{1 0}, \mathbf{1 1}]$.

Since $\cosh t$ increases with $|t|$ on the interval of integration, the possible minima of $\mathcal{R}(z \cosh t)$ occur at either $t=w$ or $t=0$. Hence, the minimum may be at an endpoint or an interior point.

When $w>0$ there is no interior saddle point and $t=0$ is irrelevant. The endpoint $t=w$ is the contributor and

$$
K_{\nu}(z, w) \sim \mathrm{e}^{-z \cosh w}\left(\frac{b_{0}}{z}+\frac{b_{1}}{z^{2}}+2 ! \frac{b_{2}}{z^{3}}+\cdots\right)
$$

where

$$
\begin{gathered}
b_{0}=\frac{\cosh \nu w}{\sinh w}, \quad b_{1}=\frac{\nu \sinh \nu w-\operatorname{coth} w \cosh \nu w}{\sinh ^{2} w}, \\
b_{2}=\frac{\nu^{2} \cosh \nu w \sinh ^{2} w-3 \nu \cosh w \sinh w \sinh \nu w+\left(2 \cosh ^{2} w+1\right) \cosh \nu w}{2 \sinh ^{5} w} .
\end{gathered}
$$

When $w=0$, the saddle point coincides with an endpoint. So

$$
K_{\nu}(z, 0) \sim \mathrm{e}^{-z}\left(\frac{\pi}{2 z}\right)^{1 / 2}\left(1+\frac{4 \nu^{2}-1}{8 z}+\cdots\right) .
$$

When $w<0$, there is an interior saddle point. Hence,

$$
K_{\nu}(z, w) \sim \mathrm{e}^{-z}\left(\frac{2 \pi}{z}\right)^{1 / 2}\left(1+\frac{4 \nu^{2}-1}{8 z}+\cdots\right) .
$$

A transition formula which indicates how (7.2) switches to (7.5) as $w$ approaches the origin is desirable. For the convenience of the reader, a method of deriving a transition formula is described in the appendix. The expansion (A 1) in the appendix leads to

$$
K_{\nu}(z, w) \sim\left(\frac{\pi}{2 z}\right)^{1 / 2} \mathrm{e}^{-z}\left(\alpha_{0}+\frac{\alpha_{1}}{z}\right) \operatorname{erfc}\left\{(2 z)^{1 / 2} \sinh \left(\frac{1}{2} w\right)\right\}+\left(\frac{\beta_{0}}{z}+\frac{\beta_{1}}{z^{2}}\right) \mathrm{e}^{-z \cosh w},
$$

where, from (A 2)-(A 5),

$$
\begin{aligned}
& \alpha_{0}=1, \quad \beta_{0}=\frac{\cosh \nu w}{\sinh w}-\frac{1}{2 \sinh \left(\frac{1}{2} w\right)}, \quad \alpha_{1}=\frac{1}{8}\left(4 \nu^{2}-1\right), \\
& \beta_{1}=\frac{\nu \sinh \nu w}{\sinh ^{2} w}-\frac{\cosh \nu w \cosh w}{\sinh ^{3} w}+\frac{1}{8 \sinh ^{3}\left(\frac{1}{2} w\right)}-\frac{4 \nu^{2}-1}{16 \sinh \left(\frac{1}{2} w\right)} .
\end{aligned}
$$


As a check on (7.7) note that, as $w \rightarrow 0, \beta_{0}$ and $\beta_{1}$ become zero, as may be seen easily from (A 6) and (A 7). Also, $\operatorname{erfc}(0)=1$ so that (7.7) is consistent with (7.5). Moreover, when $w>0$ and $\left(2 z^{2}\right)^{1 / 2} \sinh \left(\frac{1}{2} w\right)$ is large, the asymptotic expansion

$$
\operatorname{erfc} z \sim \frac{\mathrm{e}^{-z^{2}}}{\pi^{1 / 2} z}\left\{1+\sum_{p=1}^{\infty} \frac{\left(p-\frac{1}{2}\right) !(-1)^{p}}{\pi^{1 / 2} z^{2 p}}\right\}, \quad|\operatorname{ph} z|<\frac{3}{4} \pi,
$$

can be employed. Then the first two terms of (7.2) are recovered from (7.7). For $w<0$ and $\left|\left(2 z^{2}\right)^{1 / 2} \sinh \left(\frac{1}{2} w\right)\right|$ large, the relation

$$
\operatorname{erfc}(-z)=2-\operatorname{erfc}(z)
$$

leads to (7.6).

\section{Large order}

The asymptotics of $K_{\nu}(z, w)$ when the magnitude of the order is large are somewhat complex. Some idea of the behaviour can be gained by setting $\nu=z \sinh \alpha$ with the simplification that $\alpha>0$. The restriction $|\mathrm{ph} z|<\pi / 2$ is still imposed; it implies that $|\operatorname{ph} \nu|<\pi / 2$ on account of the reality of $\alpha$.

The real part of the exponent

$$
z \cosh t \mp \nu t=\nu(\cosh t \operatorname{cosech} \alpha \mp t)
$$

possesses a minimum at $t= \pm \alpha$. However, the upper sign always leads to smaller values than the lower sign. Therefore, it will be sufficient to confine our attention to the upper sign, i.e.

$$
K_{\nu}(z, w) \sim \frac{1}{2} \int_{w}^{\infty} \mathrm{e}^{-\nu(\cosh t \operatorname{cosech} \alpha-t)} \mathrm{d} t
$$

as $|\nu| \rightarrow \infty$ with $\alpha$ fixed.

When $w>\alpha$ the saddle point is irrelevant and

$$
K_{\nu}(z, w) \sim \frac{1}{2 \nu} \frac{\mathrm{e}^{-\nu(\cosh w \operatorname{cosech} \alpha-w)}}{\sinh w \operatorname{cosech} \alpha-1}\left\{1-\frac{\cosh w \operatorname{cosech} \alpha}{\nu(\sinh w \operatorname{cosech} \alpha-1)^{2}}+\cdots\right\} .
$$

For $w=\alpha$,

$$
\begin{aligned}
K_{\nu}(z, \alpha) \sim \frac{1}{2} \mathrm{e}^{-\nu(\operatorname{coth} \alpha-\alpha)}\left(\frac{\pi}{2 \nu \operatorname{coth} \alpha}\right)^{1 / 2} & \\
& \times\left\{1-\frac{1}{3}\left(\frac{2}{\pi \nu}\right)^{1 / 2} \tanh ^{3 / 2} \alpha+\frac{1}{24 \nu}\left(5 \tanh ^{2} \alpha-3\right) \tanh \alpha\right\},
\end{aligned}
$$

and, for $w<\alpha$,

$$
K_{\nu}(z, \alpha) \sim \mathrm{e}^{-\nu(\operatorname{coth} \alpha-\alpha)}\left(\frac{\pi}{2 \nu \operatorname{coth} \alpha}\right)^{1 / 2}\left\{1+\frac{1}{24 \nu}\left(5 \tanh ^{2} \alpha-3\right) \tanh \alpha\right\} .
$$


An expression to cope with the transition as $w$ passes through $\alpha$ is

$$
\begin{aligned}
K_{\nu}(z, \alpha) \sim \frac{1}{2}\left(\alpha_{0}+\frac{\alpha_{1}}{\nu}\right)\left(\frac{\pi}{2 \nu}\right)^{1 / 2} \operatorname{erfc}\{ & \left.-b\left(\frac{\nu}{z}\right)^{1 / 2}\right\} \mathrm{e}^{-\nu(\operatorname{coth} \alpha-\alpha)} \\
& +\frac{1}{2 \nu}\left(\beta_{0}+\frac{\beta_{1}}{\nu}\right) \mathrm{e}^{-\nu(\cosh w \operatorname{cosech} \alpha-w)}
\end{aligned}
$$

where

$$
\begin{gathered}
\alpha_{0}=\frac{1}{(\operatorname{coth} \alpha)^{1 / 2}}, \quad \alpha_{1}=\frac{\left(5 \tanh ^{2} \alpha-3\right)(\tanh \alpha)^{3 / 2}}{24}, \\
\beta_{0}=\frac{1}{\sinh w \operatorname{cosech} \alpha-1}+\frac{\alpha_{0}}{b} \\
\beta_{1}=-\frac{\cosh w \operatorname{cosech} \alpha}{(\sinh w \operatorname{cosech} \alpha-1)^{3}}-\frac{\alpha_{0}}{b^{3}}+\frac{\alpha_{1}}{b}
\end{gathered}
$$

and

$$
b= \pm 2^{1 / 2}(\cosh w \operatorname{cosech} \alpha-w-\operatorname{coth} \alpha+\alpha)^{1 / 2}
$$

when $w \lessgtr \alpha$. In the limit as $w \rightarrow \alpha$

$$
\beta_{0}=-\frac{1}{3} \tanh ^{2} \alpha, \quad \beta_{1} \rightarrow \frac{4}{15} \tanh ^{3} \alpha-\frac{8}{27} \tanh ^{5} \alpha .
$$

Note that it is not permissible to allow $\alpha$ to tend to zero without a more careful examination of the contribution of that part of the integral which was neglected in (8.1).

\section{Appendix A.}

This appendix sets out a method for determining the transitional asymptotic behaviour as a saddle point and endpoint approach one another. It will be sufficient for our purposes to discuss

$$
I+\int_{c}^{\infty} \mathrm{e}^{-z p(t)} q(t) \mathrm{d} t
$$

with real $c$ and non-singular $q(t)$. The function $p(t)$ will be taken to be real and to possess a single simple saddle point at $t=a$ in the interval of interest; $a$ may be greater or less than $c$. It will be assumed that $p(t)$ has a minimum at the saddle point so that $p^{\prime}(a)=0$ and $p^{\prime \prime}(a)>0$. It will also be supposed that $|\operatorname{ph} z|<\pi / 2$.

Make the substitution

$$
p(t)-p(a)=\frac{1}{2}(u-b)^{2}
$$

so that $u=b$ corresponds to $t=a$. Take $u>b$ for $t>a$ and $u<b$ for $t<a$. Choose $b$ so that $u=0$ corresponds to $t=c$, while $b$ has the same sign as $a-c$. Hence,

$$
b= \pm 2^{1 / 2}\{p(c)-p(a)\}^{1 / 2}
$$

when $a \gtrless c$. 
After the substitution

$$
I \mathrm{e}^{z p(a)}=\int_{0}^{\infty} g_{0}(u) \mathrm{e}^{-z(u-b)^{2} / 2} \mathrm{~d} u,
$$

where

$$
g_{0}(u)=q(t) \frac{\mathrm{d} t}{\mathrm{~d} u}
$$

Let

$$
g_{0}(u)=\alpha_{0}+\beta_{0}(u-b)+u(u-b) G_{0}(u)
$$

Then

$$
I \mathrm{e}^{z p(a)}=\alpha_{0}\left(\frac{\pi}{2 z}\right)^{1 / 2} \operatorname{erfc}\left\{-b\left(\frac{1}{2} z\right)^{1 / 2}\right\}+\frac{\beta_{0}}{z} \mathrm{e}^{-b^{2} z / 2}+\int_{0}^{\infty} u(u-b) G_{0}(u) \mathrm{e}^{-z(u-b)^{2} / 2} \mathrm{~d} u,
$$

where

$$
\operatorname{erfc}(w)=\frac{2}{\pi^{1 / 2}} \int_{w}^{\infty} \mathrm{e}^{-y^{2}} \mathrm{~d} y .
$$

Integration by parts yields

$$
\int_{0}^{\infty} u(u-b) G_{0}(u) \mathrm{e}^{-z(u-b)^{2} / 2} \mathrm{~d} u=\frac{1}{z} \int_{0}^{\infty} g_{1}(u) \mathrm{e}^{-z(u-b)^{2} / 2} \mathrm{~d} u
$$

with

$$
g_{1}(u)=G_{0}(u)+u G_{0}^{\prime}(u) .
$$

Repeat the above procedure by setting

$$
g_{1}(u)=\alpha_{1}+\beta_{1}(u-b)+u(u-b) G_{1}(u),
$$

which adds $\alpha_{1} / z$ and $\beta_{1} / z$ to $\alpha_{0}$ and $\beta_{0}$, respectively. Clearly, further repetition is feasible and

$$
I \sim\left(\alpha_{0}+\frac{\alpha_{1}}{z}+\cdots\right)\left(\frac{\pi}{2 z}\right)^{1 / 2} \operatorname{erfc}\left\{-b\left(\frac{1}{2} z\right)^{1 / 2}\right\} \mathrm{e}^{-z p(a)}+\left(\beta_{0}+\frac{\beta_{1}}{z}+\cdots\right) \frac{\mathrm{e}^{-z p(c)}}{z} .
$$

The coefficients in (A 1) are given by

$$
\begin{aligned}
& \alpha_{0}=g_{0}(b)=\frac{q(a)}{\left\{p^{\prime \prime}(a)\right\}^{1 / 2}}, \\
& \beta_{0}=\frac{q(c)}{p^{\prime}(c)}+\frac{q(a)}{b\left\{p^{\prime \prime}(a)\right\}^{1 / 2}}
\end{aligned}
$$

and

$$
\alpha_{1}=\frac{\frac{1}{2} q^{\prime \prime}\left(p^{\prime \prime}\right)^{2}-\frac{1}{2} q^{\prime} p^{\prime \prime \prime} p^{\prime \prime}-\frac{1}{8} q p^{(\mathrm{iv})} p^{\prime \prime}+\frac{5}{24} q\left(p^{\prime \prime \prime}\right)^{2}}{\left(p^{\prime \prime}\right)^{7 / 2}},
$$


the derivatives being evaluated at $t=a$. In addition,

$$
\beta_{1}=\frac{q^{\prime}(c)}{\left\{p^{\prime}(c)\right\}^{2}}-\frac{q(c) p^{\prime \prime}(c)}{\left\{p^{\prime}(c)\right\}^{3}}-\frac{\alpha_{0}}{b^{3}}+\frac{\alpha_{1}}{b} .
$$

When $c \rightarrow a$, limits of (A 3) and (A 5) are required. They are

$$
\beta_{1} \rightarrow \frac{\left\{\begin{array}{c}
q_{0} \\
q^{\prime \prime \prime}\left(p^{\prime \prime}\right)^{3}-2 q^{\prime \prime} p^{\prime \prime \prime}\left(p^{\prime \prime}\right)^{2}+\frac{q p^{\prime \prime \prime}}{3\left(p^{\prime \prime}\right)^{2}}, \\
q^{\prime} p^{\prime \prime}\left(p^{\prime \prime \prime}\right)^{2}-\frac{8}{9} q\left(p^{\prime \prime \prime}\right)^{3} \\
\left.-q^{\prime} p^{(\mathrm{iv})}\left(p^{\prime \prime}\right)^{2}+q p^{\prime \prime} p^{\prime \prime \prime} p^{(\mathrm{iv})}-\frac{1}{5} q p^{(\mathrm{v})}\left(p^{\prime \prime}\right)^{2}\right\}
\end{array}\right.}{3\left(p^{\prime \prime}\right)^{5}}
$$

evaluated at $t=a$.

\section{Note added in proof}

Cicchetti and Faraone [4] define their incomplete Bessel functions by (2.4) with the restriction $|\operatorname{ph} z|<\pi / 2$ and by its analogue for Hankel functions with $\operatorname{ph} z$ adjusted accordingly. For these three functions, the authors find asymptotic expressions as $z \rightarrow \infty$ when $\nu$ is real, while $|\mathcal{I} w|<\pi / 2$. On the common ground where $\nu$ and $w$ are both real and $z \rightarrow \infty$, the authors' asymptotic formula and (7.7) agree.

\section{References}

1. M. M. AGREst AND M. S. MAKSimov, Theory of incomplete cylinder functions and their applications (Springer, 1971).

2. N. Bleistein and R. A. Handelsman, Asymptotic expansions of integrals (Holt, Rinehart and Winston, 1975).

3. D. C. Chang AND R. J. Fisher, A unified theory on radiation of a vertical electric dipole above a dissipative earth, Radio Sci. 9 (1974), 1129-1138.

4. R. Cicchetti and A. Faraone, Incomplete Hankel and modified Bessel functions: a class of special functions for electromagnetics, IEEE Trans. Antennas Propagat. AP 52 (2004), 3373-3389.

5. S. L. Dvorak, Applications for incomplete Lipschitz-Hankel integrals in electromagnetics, IEEE Antennas Propagat. Mag. 36 (1994), 26-32.

6. D. S. Jones, Introduction to asymptotics (World Scientific, 1997).

7. D. S. JonEs, Incomplete Bessel functions, II, , in press.

8. L. LEwIN, The near field of a locally illuminated diffracting edge, IEEE Trans. Antennas Propagat. AP 19 (1971), 134-136.

9. M. M. Mechaik and S. L. Dvorak, Exact closed form field expressions for a semiinfinite traveling-wave current filament in homogeneous space, J. Electromagn. Waves Appl. 8 (1994), 1563-1584.

10. F. W. J. Olver, Asymptotics and special functions (Academic Press, 1974).

11. R. Wong, Asymptotic approximations of integrals (Academic Press, 1989). 